\title{
Synthesis of SAPO-34 membrane using double structure directing agents
}

\author{
Haoyang Li, Xiang Yin, Mengqiu Wan, Guofeng Sun, Shengpeng Liü
}

Key Laboratory for Green Chemical Process of Ministry of Education, Hubei Key Laboratory of Novel Reactor and Green Chemical Technology, Wuhan Institute of Technology, Wuhan 430205, China

aliuabss@163.com, *Corresponding author

Keywords:SAPO-34 membrane; double templates; secondary growth; separation.

Abstract. SAPO-34 membranes were synthesized using double structure directing agents (tetraethylammoniumhydroxide (TEAOH) and dipropylamine (DPA)). Using the secondary growth method, the seeds synthesized by hydrothermal process provided nucleation for the membrane growth and accelerated the zeolite crystallization. The prepared seed crystals along with SAPO-34 membrane were characterized by X-ray diffraction (XRD) and Scanning electron microscopy (SEM). The single gas $\left(\mathrm{H}_{2}, \mathrm{~N}_{2}\right.$ and $\left.\mathrm{CO}_{2}\right)$ and mixture $\left(\mathrm{H}_{2}, \mathrm{~N}_{2}\right.$ and $\left.\mathrm{CO}_{2}\right)$ perm-selective properties were evaluated for the performance of the formed membrane. The higher $\mathrm{H}_{2} / \mathrm{CO}_{2}$ and $\mathrm{H}_{2} / \mathrm{N}_{2}$ separation factors are 5.83 and 5.01, respectively, for the SAPO-34 membrane at $298 \mathrm{~K}$ and a feed pressure of $100 \mathrm{kPa}$.

\section{Introduction}

Carbon dioxide is an impurity of hydrogen and must be removed for the hydrogen energy utilization. An energy-efficient approach to separate $\mathrm{CO}_{2}$ from $\mathrm{H}_{2}$ is through using zeolite membranes. Zeolites with uniformly sized pores of molecular dimensions used for $\mathrm{H}_{2} / \mathrm{CO}_{2}$ separations include zeolite T [1, 2], DDR [3], MFI [4] and SAPO-34 [5-8].

The secondary growth method appears to be the best studied method for the formation of SAPO34 zeolite membranes [9, 10]. In this method the zeolite seeds coated on the support surface provided nucleation for the membrane growth and accelerated the zeolite crystallization. In this study, SAPO-34 membranes were synthesized by combining structure directing agents (SDAs). Tetraethylammonium hydroxide (TEAOH) was used as the main SDA and dipropylamine (DPA) as the secondary SDA in the process of preparing the SAPO-34 membranes. The combination of double structure directing agents for membrane formation resulted in SAPO-34 membranes with a higher selectivity than previously reported [11]. The prepared seed crystals along with SAPO-34 membrane were characterized by X-ray diffraction (XRD) and Scanning electron microscopy (SEM). The single gas permeation measurement for $\mathrm{H}_{2}$ and $\mathrm{CO}_{2}$ was examined for the performance of the membranes.

\section{Experimental}

Synthesis procedure for SAPO-34 seeds: In a typicalsynthesis, aluminumiso-propoxide(Al(i$\left.\mathrm{C}_{3} \mathrm{H}_{7} \mathrm{O}\right)_{3}$, Aldrich99.99\%), TEAOH(25 wt\% aqueous solution, Sigma-Aldrich), deionized water, AS-40colloidal silica (30 wt $\%$ aqueous suspension), and $\mathrm{H}_{3} \mathrm{PO}_{4}(85 \mathrm{wt} \%$ aqueous solution, SigmaAldrich) were added one after another and stirred to form a homogeneous solution. The resulting solution was stirred for $24 \mathrm{~h}$ at room temperature. The solution was then placed in an autoclave and held at $473 \mathrm{~K}$ for $24 \mathrm{~h}$. After being washed and centrifuged, SAPO-34 seeds were obtained.

Synthesis procedure for SAPO-34 membranes: The pre-treated supports were seeded with previously synthesized SAPO-34 zeolite crystals by the dip-coating method. The seeded support was immersed into the SAPO-34 membranes gel contained in an autoclave and hydrothermal synthesis was carried out at $483 \mathrm{~K}$ for $6 \sim 8 \mathrm{~h}$. After being washed and centrifugedat $823 \mathrm{~K}$ for $6 \mathrm{~h}$, the SAPO-34 zeolite membranes were harvested. TheSAPO-34 membranes gel was prepared by adding $\mathrm{Al}\left(\mathrm{i}-\mathrm{C}_{3} \mathrm{H}_{7} \mathrm{O}\right)_{3}$ to the mixture of DI water and $\mathrm{H}_{3} \mathrm{PO}_{4}$ and stirred for $2 \mathrm{~h}$. Ludox AS-40 
colloidal silica gel, TEAOH and DPA were added to the gel one by one after stirring for $0.5 \mathrm{~h}$. After aging the resulting gel at $323 \mathrm{~K}$, the membranes gel was prepared.

Permeation measurements: Single-gas and mixture permeations were carried out using a special permeation designed by our laboratory. The gas permeance of themembrane was measured by soap film flow meter underthe feed pressure of $40-100 \mathrm{kPa}$ at room temperature. The gases testedin the membranes were $\mathrm{H}_{2}, \mathrm{~N}_{2}$ and $\mathrm{CO}_{2}$ and the driving forcewas the pressuredifference between both sides of the membranewhich was controlled using a back-pressure regulator.

Characterization: The XRD patterns were collected on a Philips 1710 diffractometer using $\mathrm{Cu} \mathrm{K} \alpha$ $(\lambda=1.542 \AA$ ) radiation at $30 \mathrm{kV}$ and $40 \mathrm{~mA}$ to determine the structural properties of the synthesized membranes and seeds. The top surface and cross-section images of the membrane layer were analyzed by Scanning electron microscopy (SEM).

\section{Results and Discussion}

The XRD patterns shown in Fig. 1a indicated that samples show the typical diffraction patterns of CHA-structure SAPO-34 without presence of other impurity phases [5].The morphologies and sizes of the SAPO-34 crystals were characterized by Scanning electron microscopy (SEM). The seed crystals were cubic with particle sizes about $300 \mathrm{~nm}$, as shown in Fig. 1 b.
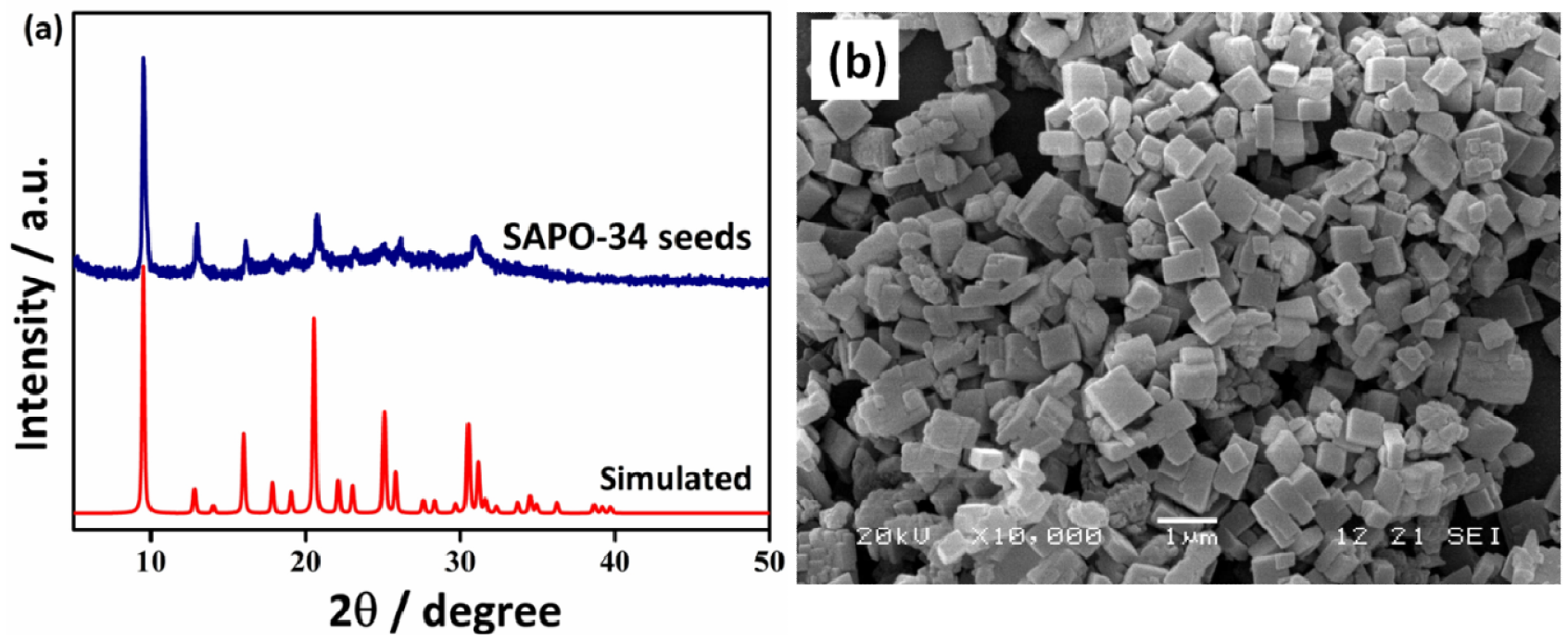

Fig. 1 a) XRD pattern of the seed crystals, b) SEM images of SAPO-34 seed crystals.

SEM images of the membrane surfaces (Fig. 2a) show well-intergrown micron range zeolite crystals for SAPO-34 membranes prepared with two structure directing agents TEAOH and DPA. Cross-section SEM images of the membranes (Fig. 2b) show that a dense zeolite layer about $8 \mu \mathrm{m}$ was synthesized on porous $\mathrm{Al}_{2} \mathrm{O}_{3}$ support. The prepared membranes without any defects played a key role in the separation of gas mixture. 


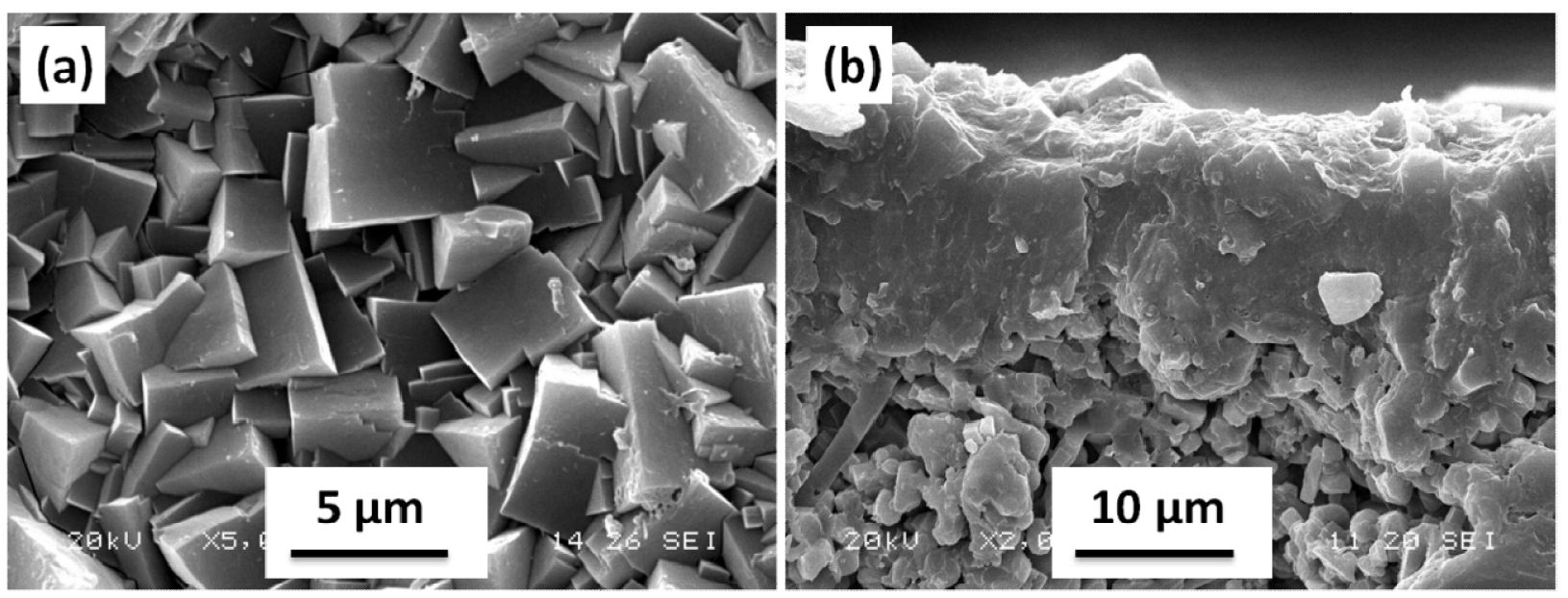

Fig. 2 Typical surface (a) and cross-section (b) SEM images of the SAPO-34 membranes prepared with two structure directing agents TEAOH and DPA.

The membranes had $\mathrm{H}_{2} / \mathrm{CO}_{2}$ separation selectivity higher than 6 , which was much higher than Knudsen coefficient $\left(\mathrm{H}_{2} / \mathrm{CO}_{2}=4.8\right)$, with $\mathrm{H}_{2}$ permeance as high as $5 \times 10^{-6} \mathrm{~mol} \cdot \mathrm{m}^{-2} \cdot \mathrm{s}^{-1} \cdot \mathrm{Pa}^{-1}$ at 298 K(shown in Fig. 3). As shown in Fig. 4, the synthesized SAPO-34 membranes also possessed a higher selectivity for $\mathrm{H}_{2} / \mathrm{N}_{2}$ separation. Note that the hydrogen permeance and the $\mathrm{H}_{2} / \mathrm{CO}_{2}$ along with $\mathrm{H}_{2} / \mathrm{N}_{2}$ separation factor for the SAPO-34 membranes synthesized using double structure direction agents were higher than those corresponding to the membrane prepared with single template TEAOH [12].

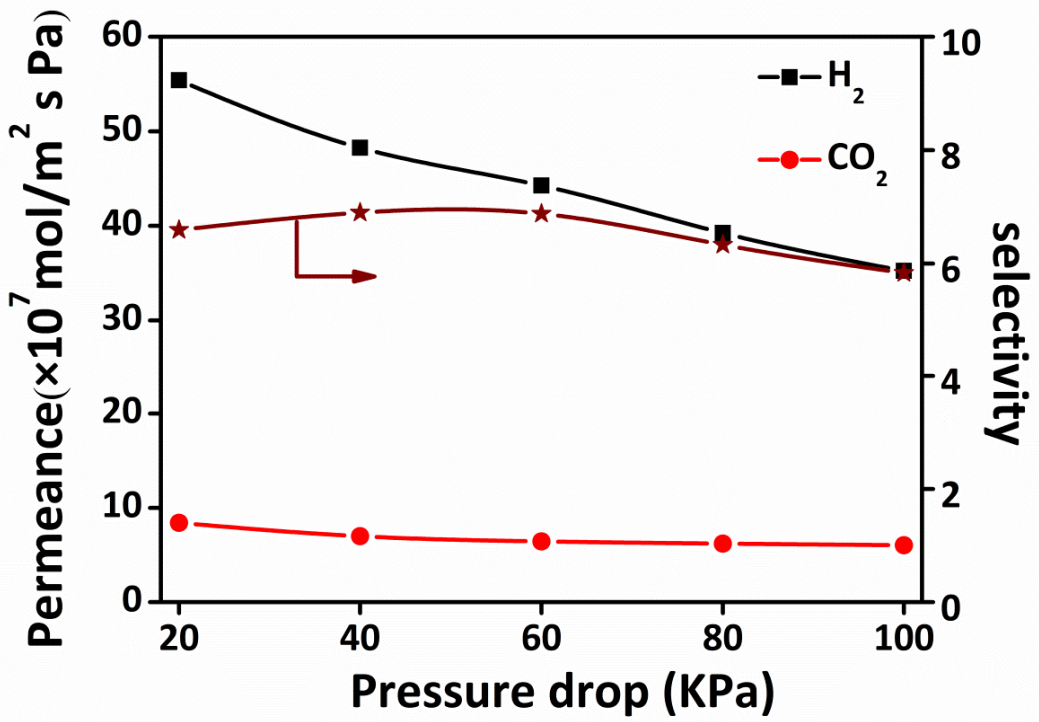

Fig. 3 Permeance and separation selectivity for $\mathrm{H}_{2} / \mathrm{CO}_{2}$ mixture at $298 \mathrm{~K}$ 


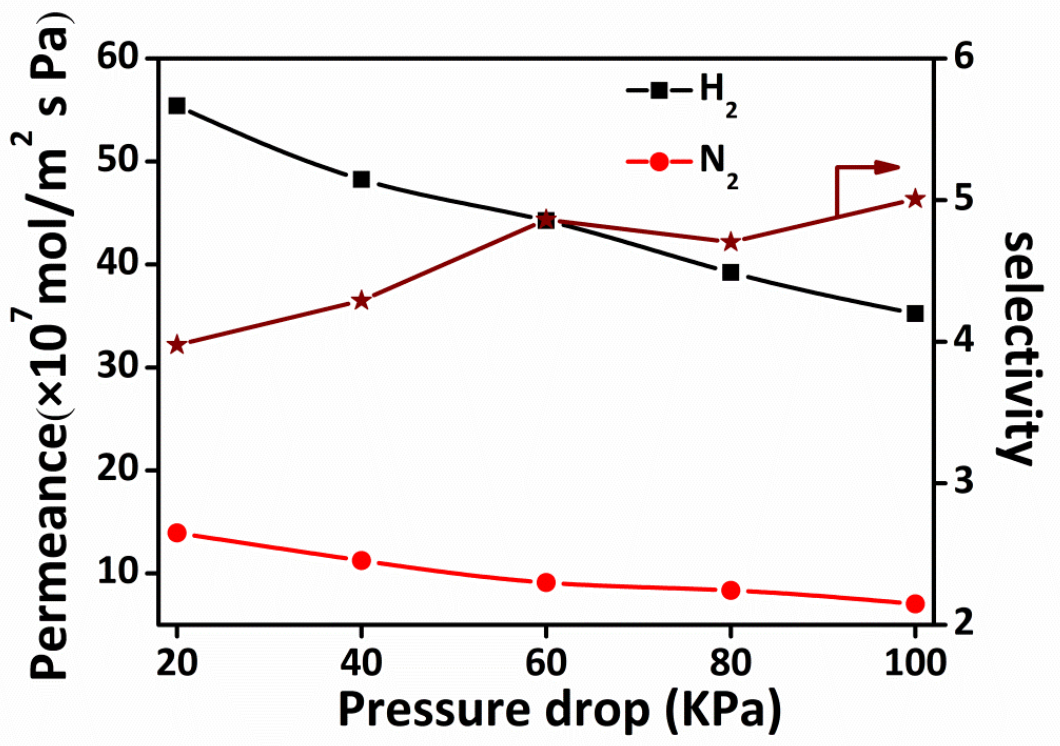

Fig. 4 permeance and separation selectivity for $\mathrm{H}_{2} / \mathrm{N}_{2}$ mixture at $298 \mathrm{~K}$

\section{Conclusions}

A dense SAPO-34 zeolite membrane without defects was synthesized using double structure direction agents, TEAOH and DPA. The membranes had higher selectivity for $\mathrm{H}_{2} / \mathrm{CO}_{2}$ and $\mathrm{H}_{2} / \mathrm{N}_{2}$ separation, with $\mathrm{H}_{2}$ permeance as high as $5 \times 10^{-6} \mathrm{~mol} \cdot \mathrm{m}^{-2} \cdot \mathrm{s}^{-1} \cdot \mathrm{Pa}^{-1}$ at $298 \mathrm{~K}$ and a feed pressure of $100 \mathrm{kPa}$.

\section{Acknowledgements}

We are grateful for the financial support fromMajor Innovation Project of Science and Technology of Hubei Province (2014ACA038).

\section{References}

[1] S.M. Mirfendereski, T. Mazaheri, M. Sadrzadeh, T. Mohammadi: Sep. Purifi.Technol.Vol. 61(2008), p. 317

[2] S. Feng, T. Bein: Nature.Vol. 368 (1994), p. 834

[3] S. Himeno, T. Tomita, K. Suzuki, K. Nakayama, K. Yajima, S. Yoshida: Ind. Eng.Chem. Res.Vol. 46 (2007), p. 6989

[4] J.C. Poshusta, R.D. Noble, J.L. Falconer: J. Membr. Sci. Vol. 160 (1999), p. 115

[5] M.A. Carreon, S. Li, J.L. Falconer, R.D. Noble: Adv.Mater.Vol. 20 (2008), p. 729

[6] J.K. Das, N. Das, S. Bandyopadhyay: Int. J. Hydrogen Energy. Vol. 37 (2012), p. 10354

[7] J.K. Das, N. Das: ACS.Appl.Mater. Inter.Vol. 6 (2014),p. 20717

[8] R. Zhou, E.W. Ping, H.H. Funke, J.L. Falconer, R.D. Noble: J. Membr. Sci.Vol. 444 (2013), p. 384

[9] M.A. Carreon, S. Li, J.L. Falconer, R.D. Noble: J. Am. Chem. Soc. Vol. 130 (2008), p. 5412

[10] G. Feng, P. Cheng, W. Yan, M. Boronat, X. Li, J.H. Su, J. Wang, Y. Li, A. Corma, R. Xu: Sci. Foun. Chin.Vol. 351 (2016), p. 1188

[11] J.K. Das, N. Das, S.N. Roy, S. Bandyopadhyay: Ceram. Int.Vol. 25 (2012), p. 1699

[12] J.C. Poshusta, V.A. Tuan, E.A. Pape, R.D. Noble, J.L. Falconer: AIChE. J. Vol. 46 (2000), p. 779 